by

Gilbert Paillé ${ }^{1}$

\section{Summary}

This paper was presented at a regional forestry association meeting to a large audience of woodlot owners, small forest entrepreneurs and marketing board representatives. Its first purpose was to demonstrate that reforestation of isolated and abandoned agricultural fields is seldom economical when viewed as a stand-alone activity, because the man planting trees can seldom live long enough to harvest the crop, thus failing to recuperate any return on his investment. This is a widely accepted view.

The second purpose of the paper however was to demonstrate that forestation of a management unit (which is done either to hasten the renewal of cutovers, to put back into full production the productive acres struck by natural accidents, or to enlarge the management unit) is always a highly economical activity. It is so mainly because it allows the owner to increase immediately the harvest of mature timber on his property (Intensive Management Effect or Allowable Cut Effect), and to no longer consider the cost of plantation as a long-term investment but as a logging cost.

In such a case, a substantial portion of the money needed to finance such a desirable activity does not come from a saving account or from borrowed capital but from the realized stumpage value itself, which owner should see as a profit only after the forest has been renewed, tended and managed properly. This is not a widely known nor a widely accepted concept in Canada, but it is in Europe.

Plainly stated, it means that the forest should pay for its management. It also means that forestation is a highly economical activity not only for the owner but for the community as a whole because, on most properties, it should allow for an immediate increase in harvest, transportation, conversion and services, thus bringing more revenues and benefits to owners, entrepreneurs and governments.

\section{Introduction}

Le directeur-gérant de votre Association, M. Lucien Bédard, m'a demandé de vous entretenir expressément de la rentabilité des investissements dans le reboisement. II a insisté pour que je discute du coût des plantations, de leur rendement et du financement des travaux. II m'a même sommé de dire si oui ou non les reboisements étaient rentables au Québec.

Puisque j'ai accepté son invitation, je vais tenter de suivre son programme. Cependant, je vais d'abord vous entretenir d'aménagement forestier et tenter de vous faire voir comment le reboisement et la régénération forestière doivent être associés et combien ils sont nécessaires avant de discuter de leur rentabilité.

\section{Objectif des Plantations}

Lorsqu'un propriétaire de terrain privé parle de plantation, il pense normalement à reboiser une parcelle de terrain déboisé, cultivé pendant un certain temps et abandonné par

\footnotetext{
1Texte présenté à l'Assemblée Annuelle de l'Association Forestière des Cantons de l'Est, Magog, PQ, le 8 octobre 1981.

tons de l'Est, Magog, PQ, le 8 octobre 1981.
2 Directeur de la recherche forestière, CIP Inc., Montréal.
}

la suite pour diverses raisons. Et c'est normal, car le mot reboisement lui-même signifie reforestation ou repeuplement artificiel d'un fond autrefois boisé.

Lorsqu'un ingénieur forestier parle de plantation, il pense d'abord à l'activité qui a pour but de régénérer les parterres de coupe d'une unité d'aménagement où la régénération naturelle désirable ne se produit pas assez vite, ou n'est pas susceptible de s'installer faute de lits de germination propices, faute de sources de semences adéquates ou faute de soins appropriés apportés avant ou pendant la récolte de la forêt mûre. II pense ensuite à regarnir les aires déboisées dans l'unité par les accidents naturels comme le feu, les insectes et les maladies. II pense en fait à garder les terrains forestiers productifs en production pour assurer la pérennité de la forêt. II pense en termes de forestation d'une unité plutôt que de reforestation d'une parcelle isolée de terre dénudée.

II peut aussi penser en termes d'agrandissement de la superficie forestière productive aménagée principalement pour la production de matière ligneuse. Cet agrandissement peut inclure la récupération de certains terrains improductifs, peu productifs ou temporairement "sans vocation" bien définie, telles les terres abandonnées dans les zones agricoles en régression.

La différence entre ces deux approches est considérable et fondamentale. En effet, le fait de hâter la régénération des parterres de coupe, de remettre rapidement en production les aires productives avariées et d'agrandir l'unité d'aménagement en récupérant des terrains à potentiel forestier permet la plupart du temps une augmentation immédiate de la récolte des forêts mûres situées dans la même unité. Cette augmentation de l'activité de récolte et de production augmente les activités de transport, d'usinage et de services. Elle augmente par conséquent les revenus des propriétaires fonciers, des industriels et des gouvernements.

Cet effet multiplicateur de la simple activité de plantation est celui qui en fait un traitement excessivement rentable dans la majorité des cas. On pourrait l'appeler EAl ou Effet d'Aménagement Intensif.

Par contre, lorsque le reboisement n'est vu que comme un placement à long terme dans le but de regarnir ou d'embellir un champ devenu inutile, il est extrêmement rare qu'il puisse être considéré comme une activité rentable, du moins au niveau du propriétaire, parce qu'il lui faudra attendre trop longtemps pour réaliser le surplus de revenus escompté.

Donc, pour être rentables, les plantations doivent d'abord faire partie intégrante de la stratégie d'aménagement d'une propriété forestière. Elles doivent être effectuées en vue d'accélérer la régénération de la forêt ou d'agrandir la superficie forestière productive de la propriété. Elles doivent permettre une augmentation immédiate ou prochaine de l'activité de récolte de la forêt mûre. Telles sont les conditions de base de la rentabilité des plantations.

Par ailleurs, puisqu'elles occasionnent des déboursés assez considérables, les plantations doivent être effectuées de la façon la plus économique possible et seulement aux endroits où elles sont nécessaires. 
Avant de procéder aux calculs de coûts et revenus, voyons d'abord si les plantations sont nécessaires dans les Cantons de l'Est.

\section{Besoin des Plantations}

Un examen des statistiques forestières récentes (Barrette et Langevin, 1979) montre que les efforts de régénération forestière devraient être déculpés dans les forêts privées des Cantons de l'Est. Ceci permettrait de remettre rapidement en production tous les parterres de coupe et aussi de récupérer au cours d'une période raisonnable les quelque 150000 acres de friches et de vieux bûchés mal régénérés ou en voie de se régénérer en espèces de peu de valeur commerciale.

Pour ce faire, il faudrait que tous les producteurs de bois, entrepreneurs et industriels forestiers de la région (environ 2000 ) consacrent une semaine de travail par année à cultiver la forêt et à planter des arbres sur les territoires dont ils tirent une bonne part ou la majorité de leurs revenus. Ce serait encore mieux si tous les propriétaires de forêts privées de la région (environ 9000 ) s'y mettaient, car i ne leur faudrait alors qu'une journée de travail par année pour accomplir la même tâche.

L'activité économique générée par la récolte de quelque 250000 cordes de bois par an dans la région est considérable et doit être maintenue ou augmentée. Pour ce faire, il est nécessaire que tous les parterres de coupe restent productifs et que toutes les friches situées dans les zones agricoles considérées en régression par le Ministère de l'Agricultue soient reboisées.

Quel serait le coût d'une telle entreprise?

\section{Coût des Plantations}

Le coût des plantations varie énormément selon les difficultés de préparation du terrain, le type de semis utilisé, les méthodes employées, l'ampleur des projets et les arrangements administratifs particuliers à chacun.

Ainsi un propriétaire qui demande et reçoit gratuitement ses plants du Ministère de l'Energie et des Ressources, qui les met lui-même en terre manuellement sans préparer le terrain et sans compter son temps, peu s'imaginer que ses plantations ne coûtent rien.

Par ailleurs, on peut considérer le cas du propriétaire qui ferait préparer le terrain adéquatement, qui achèterait ses plants à la pépinière et qui paierait des ouvriers pour les mettre en terre et voir à ce qu'ils ne soient pas étouffés par la compétition au cours des premières années suivant la plantation. Pour installer 1000 semis par acre, il lui en coûterait de $\$ 30$ à $\$ 50$ pour la préparation du site, de $\$ 60$ à $\$ 130$ pour les semis rendus au lieu de la plantation, de $\$ 40$ à $\$ 100$ pour les faire planter et $\$ 10$ à $\$ 20$ pour administrer le projet; l'entretien de la plantation au cours des trois premières années pourrait représenter entre $\$ 10$ et $\$ 150$ l'acre. Au total, le coût réel serait donc de $\$ 150$ à $\$ 450$ l'acre selon les difficultés rencontrées et les méthodes employées.

Si l'etat fournissait gratuitement les semis, les frais se trouveraient réduits de $\$ 50$ à $\$ 110$ l'acre; si l'entretien devenait inutile, il y aurait aussi une économie de $\$ 10$ à $\$ 150$ l'acre. Ainsi, dans les meilleures conditions possibles, il devrait débourser de sa poche entre $\$ 90$ et $\$ 190$ l'acre pour faire planter 1000 semis. Comment devrait-il financer cette activité?
Tableau 1. Coûts des Plantations

\section{A - Coût selon la méthode}

\begin{tabular}{|c|c|c|c|c|c|c|c|c|}
\hline \multirow{2}{*}{\multicolumn{2}{|c|}{ Préparation du terrain }} & \\
\hline & & \multicolumn{3}{|c|}{3} & \multirow{2}{*}{$\begin{array}{l}\text { à } \\
\text { à }\end{array}$} & \multirow{2}{*}{\multicolumn{2}{|c|}{$\begin{array}{l}5 \\
5\end{array}$}} & \multirow[t]{2}{*}{ 一 } \\
\hline & sous-total & & 3 & & & & & \\
\hline \multirow{3}{*}{\multicolumn{2}{|c|}{$\begin{array}{l}\text { Achat de semis } \\
\text { racines nues } \\
\text { en conteneurs }\end{array}$}} & & & & & & & \\
\hline & & 4 & & & à & 6 & & \\
\hline & & & & 6 & à & & & 9 \\
\hline \multicolumn{9}{|c|}{ Transport de semis } \\
\hline $\begin{array}{l}\text { sur route } \\
\text { sur site }\end{array}$ & & & 1 & & à & & 2 & \\
\hline sur site & & & 1 & & à & & 2 & \\
\hline \multicolumn{2}{|c|}{ Mise en terre des semis } & & 4 & & à & & 10 & \\
\hline & sous-total & 10 & - & 12 & à & 20 & - & 23 \\
\hline \multicolumn{2}{|c|}{$\begin{array}{l}\text { Entretien du site } \\
\text { chimique } \\
\text { mécanique }\end{array}$} & 1 & & 10 & $\begin{array}{l}\text { à } \\
\text { à }\end{array}$ & 5 & . & 15 \\
\hline & sous-total & 1 & - & 10 & à & 5 & - & 15 \\
\hline \multirow[t]{3}{*}{ Administration } & & & 1 & & à & & 2 & \\
\hline & sous-total & & 1 & & à & & 2 & \\
\hline & total & 15 & - & 26 & à & 32 & - & 45 \\
\hline \multicolumn{5}{|c|}{$\begin{array}{c}\text { - Coût selon l'espacement } \\
800 \text { semis/acre } \\
1000 \text { semis/acre } \\
1200 \text { semis/acre }\end{array}$} & \multicolumn{4}{|c|}{$\begin{array}{c}\text { (\$/acre) } \\
120-360 \\
150-450 \\
180-540\end{array}$} \\
\hline
\end{tabular}

\section{Financement des Plantations}

Une propriété forestière aménagée pour la production de matière ligneuse devrait normalement fournir l'argent nécessaire à sa régénération. C'est dire que la valeur sur pied des bois exploités devrait être assez grande pour couvrir les frais de régénération et pour fournir au propriétaire un revenu raisonnable.

Prenons l'exemple du producteur moyen de l'Estrie qui mettrait en marché 125 cordes de bois à pâte résineux cette année. Après avoir défrayé ses coûts d'exploitation, de mise en marché, et d'administration, il serait susceptible de recevoir $\$ 1250.00$ qui représenteraient la valeur sur pied de ses bois. Pour réaliser cette valeur, il lui aurait probablement fallu couper au moins 10 acres de forêt mûre qu'il devrait voir à régénérer au plus tôt. Après examen de la situation, il pourrait décider de laisser 6 acres se régénérer naturellement et de planter 4 acres au coût de quelque $\$ 600$. Son revenu réel dans ce cas serait alors de $\$ 650$ à moins qu'il ne dónide d'en utiliser une tranche supplémentaire pour rebois ${ }^{\prime}$ s un ancien parterre de coupe qui ne se comporterait pas comme prévu, ou pour changer la composition de peuplements à maturité, mais dont la valeur sur pied des bois serait très faible ou nulle.

Si par contre aucune récolte commerciale n'était possible immédiatement sur sa propriété, ou que le volume de la récolte possible soit insuffisant pour couvrir tous les frais de régénération, le propriétaire pourrait décider de puiser dans ses économies, ou d'avoir recours au Crédit Forestier duquel il pourrait obtenir jusqu'à $\$ 40000$ à un taux d'intérêt de $5 \%$ inférieur aux taux courants pour une période de 60 ans. Cette solution l'obligerait à suivre les prescriptions d'un plan de gestion et à administrer son boisé de façon à ce qu'il lui fournisse un revenu constant et intéressant dans les meilleurs délais. Les revenus nets de la vente des bois mis en marché devraient par la suite être utilisés en partie pour amortir la dette et en partie pour assurer la reproduction rapide des forêts récoltées ou endommagées.

En somme, le bois récolté devrait payer pour l'aménagement, et les coûts de régénération de la forêt aménagée ne devraient pas être considérés comme un investissement à long terme, mais comme un coût d'exploitation de la propriété forestière. L'activité de régénération artificielle 
devrait être augmentée aussi longtemps qu'elle permet au propriétaire de boisé de profiter de l'Effet d'Aménagement Intensif, c'est-à-dire de couper plus de bois de valeur commerciale maintenant.

\section{Rendement des Plantations}

Nous avons vu jusqu'ici que les plantations produisent un Effet d'Aménagement Intensif (EAI) parce qu'elles permettent d'agrandir instantanément la base productive de l'unité d'aménagement et parce qu'elles permettent de raccourcir le délai de régénération des parterres de coupe. Une autre tranche de cet Effet peut aussi provenir d'une augmentation du rendement car, si les plantations sont bien faites, leur rendement peut être beaucoup plus élevé que celui des peuplements forestiers naturels.

Dans les Cantons de l'Est, les peuplements naturels qui sont récoltés maintenant contiennent en moyenne 12 cordes de bois à l'acre. La plupart de ces peuplements sont mûrs c'est-à-dire qu'ils ont atteint au moins 50 à 70 ans selon l'espèce et la qualité des stations. Donc, ils ont produit en moyenne 0.2 corde de bois à l'acre par année durant leur existence.

Par comparaison, les plantations de pin rouge au Québec peuvent fournir deux fois plus de bois en deux fois moins de temps, c'est-à-dire qu'elles sont quatre fois plus productives; les plantations de pin gris sont 2.5 fois plus productives; l'épinette blanche est 2 fois plus productive et l'épinette de Norvège 3 fois plus productive que la moyenne des peuplements naturels. En général, on peut donc dire que, chaque fois qu'on reboise une acre avec l'une ou l'autre de ces espèces, on peut au moins doubler la productivité naturelle de la station. Dans le cas du pin gris et de l'épinette, il serait possible d'augmenter encore cette productivité d'au moins 15 pour cent si on utilisait du stock amélioré génétiquement.

L'Effet d'Aménagement Intensif attribuable aux plantations peut donc être considérable dans les Cantons de l'Est. Mais, est-ce que l'activité nécessaire pour l'obtenir est rentable?

\begin{tabular}{|c|c|c|c|c|}
\hline Peuplement & Site & $\begin{array}{l}\text { Age } \\
\text { (an) }\end{array}$ & $\begin{array}{l}\text { Rendement } \\
\text { (cordelacre) }\end{array}$ & $\begin{array}{l}\text { Accroissement } \\
\text { annuel moyen } \\
\text { (cordelacrelan) }\end{array}$ \\
\hline \multicolumn{5}{|c|}{$\begin{array}{l}1 \text { - Forêt naturelle } \\
\text { Cantons de l'Est }\end{array}$} \\
\hline & Pauvre & $50-70$ & 6 & 0.1 \\
\hline & Moyen & $50-70$ & 12 & 0.2 \\
\hline & Bon & $50-70$ & 24 & 0.4 \\
\hline \multicolumn{5}{|l|}{2 - Plantation ${ }^{1}$} \\
\hline Pin rouge & Pauvre & 30 & 15 & 0.5 \\
\hline & Bon & 30 & 36 & 1.2 \\
\hline Pin gris & Pauvre & 30 & 8 & 0.3 \\
\hline & Bon & 30 & 24 & 0.8 \\
\hline Epinette & Pauvre & 30 & 8 & 0.3 \\
\hline blanche & Bon & 30 & 18 & 0.6 \\
\hline Epinette & Pauvre & 30 & 9 & 0.3 \\
\hline Norvège & Bon & 30 & 26 & 0.9 \\
\hline
\end{tabular}

${ }^{1}$ Tiré de: Castonguay (1977), Brillant et al. (1977). Rendements de plantations à espacement de $2,5 \times 2,5 \mathrm{~m}$

\section{Rentabilité des Plantations}

On pourrait reformuler cette question en se demandant plutôt: est-ce qu'il est rentable pour le propriétaire forestier de bien aménager son domaine boisé ou de bien gérer ses affaires?

S'il n'apporte aucune attention à la régénération rapide de la forêt qu'il exploite avec des espèces commerciales, le propriétaire s'aperçoit bientôt que la quantité et la valeur des produits forestiers qu'il peut extraire de sa pronriété diminue. Son revenu diminue et il paye moins de taxes et d'impôts. L'activité économique générée par l'utilisation des produits de sa propriété diminue aussi. Et, lorsque des milliers de producteurs se retrouvent comme lui dans l'impossibilité physique d'extraire de leur domaine forestier des produits de qualité, en quantité suffisante pour permettre à l'industrie forestière de s'approvisionner à un coût raisonnable et de produire en quantité suffisante pour répondre à la demande, c'est l'économie du pays qui en souffre gravement.

Si la négligence des propriétaires forestiers a été telle et pendant tellement longtemps qu'ils ne peuvent plus profiter de l'Effet d'Aménagement Intensif lorsqu'ils décident enfin de prendre leurs affaires en main, alors les investissements nécessaires sont tellement grands et les rendements sont tellement faibles que l'Etat doit intervenir pour leur permettre de remettre leur propriété en valeur et d'attendre que les arbres deviennent assez gros pour être utilisables et générer à nouveau les revenus nécessaires pour assurer leur pérennité. Les politiciens qui doivent prendre ces décisions économiques au nom de l'Etat sont réticents parce qu'ils ne peuvent pas voir ni quand ni comment ils pourront utiliser l'impact des retombées positives de ces actes. Et le cercle vicieux s'agrandit.

Heureusement, la plupart des propriétaires de boisés n'ont pas encore atteint le point de non-retour. Plusieurs ont su conserver une partie de leur avoir forestier sur pied de sorte qu'ils peuvent maintenant se permettre de bénéficier de l'Effet d'Aménagement Intensif pour rentabiliser au maximum leurs activités forestières. Ils peuvent utiliser la théorie des investissements pour comparer la rentabilité financière de plusieurs stratégies d'aménagement (Schweitzer and Schuster, 1976) et choisir la meilleure. Ils peuvent même se regrouper avec d'autres propriétaires à l'intérieur des Groupements Forestiers pour agrandir l'unité de gestion, bénéficier d'un plus grand Effet d'Aménagement Intensif et de l'aide de l'Etat pour la remise en valeur des terres forestières du Québec habité. Nul doute que dans ce cadre, l'activité de reboisement est autant sinon plus rentable que la plupart des autres activités économiques qui ont pour objet la mise en valeur des ressources naturelles renouvelables.

\section{Conclusion}

En conclusion, nous pouvons affirmer que les plantations sont rentables pour le propriétaire lorsqu'elles lui permettent de bénéficier de l'Effet d'Aménagement Intensif. II faut planter des arbres pour accélérer la régénération des aires de coupe, pour agrandir la superficie forestière productive ou pour augmenter le rendement des peuplements de la propriété aménagée pour la production soutenue de matière ligneuse. Le coût des plantations dans ces conditions doit être considéré comme un coût d'exploitation de la matière ligneuse; il doit être traité comme une dépense annuelle d'opération de la propriété forestière (déductible d'impôt) et non comme un investissement à long terme dont on ne pourra récupérer les intérêts que longtemps après sa mort.

Cette activité doit être financée par les revenus de l'exploitation de la forêt mûre. Si la forêt mûre ne peut suffire à générer assez de revenus pour ce faire, c'est qu'on en a abusé dans le passé; il faut alors emprunter pour faire le traitement et laisser à la forêt le temps de s'équilibrer. L'Etat dans ce cas peut consentir à donner un coup de main. Cependant, il faut comprendre que les meilleures plantations du monde ne feront pas vieillir les peuplements actuels plus vite.

Il faut planter pour aménager. Telle est la loi de la rentabilité des plantations.

\section{References}

Barrette, Y. et D. Langevin. 1979. Ressources et activités en forêt privée. Dir. Gen. For., Min. Terres et Forêts. 144 p. 
Brillant, C. et al. 1977. Rentabilité des reboisements au Québec. Service de la Rech., Dir. Gén. Forêts, MTF. Mémoire No 36. 179 p.

Castonguay, A. 1977. Rentabilité des reboisements au Québec Service de la Rech., Dir. Gén. Forêts, MTF. Mémoire No 38. 166 p.

Paillé, G. 1969. Considérations sur le partage des coûts et investissements en aménagement des forêts. Opérations
Forestières, Juin. 32-38.

Paillé, G. 1976. L'aménagement des forêts privées du Québec. Le Fonds de Recherches Forestières de I'Université Laval, Bulletin No $14,129 \mathrm{p}$.

Schweitzer, D.L. and E.G. Schuster. 1977. Economics and tree planting in the Inland Northwest. Washington State U. Coop. Extension Service. $11 \mathrm{p}$.

\title{
The Evolution and Status of Canadian Silviculture Practice
}

by

\author{
G.F. Weetman 1
}

\begin{abstract}
The development of Canadian silviculture practice is outlined. Five development stages are identified. Based on a 1980 survey, corporate silviculture activity is presented by province and by freehold ownership. The intensification of silviculture practice will be dependent on the success of provincial/federal/industrial mechanisms to put more than one nickel of every dollar generated back into the forest.
\end{abstract}

\section{Résumé}

En identifiant cinq périodes du développement, l'auteur fait l'esquisse de la pratique sylvicole au Canada. Sur la base d'un relevé de 1980, il présente par province et par mode de tenure les apports sylvicoles. L'intensification de la pratique sylvicole dépend d'un effort supérieur de réinvestissement des argents que génère la forêt de la part du provincial, du fédéral et de l'industrie.

\section{Introduction}

Historically the major challenges to Canadian foresters have been the inventory of the vast forest resources, the initiation of forest protection programmes to hold down catastrophic losses, the construction of roads for access and the logging of mature and overmature growing stock in a cost effective manner. During the period from 1920 to 1970 these activities dominated Canadian forestry practice. From 1920 onwards implementation of large scale silviculture practices, particularly regeneration and protection practices, have been the focus of much discussion, debate and revised policy and legislation. (Canadian Forestry Association 1977, Reed 1978, Can. Pulp Paper Assn. 1981).

The driving forces for this change have been the rising demand for forest products, declines in accessible, high value virgin forest available for logging, growing concern over inadequațe regeneration on cutover land and continued large

\footnotetext{
${ }^{1}$ Faculty of Forestry, The University of British Columbia, Vancouver, BC., V6T 1W5.
}

growing stock losses to insects, fire and disease.

The responses of the provincial crown land management agencies have varied in speed and detail of legislative and regulatory actions, but they have shown a common trend towards assigning increasing responsibility to industrial licencees for on the ground silviculture practices. The driving forces for this change in policy have been:

1. Difficulties in funding and implementing programmes within civil service budgeting and manpower constraints.

2. Recognition of the value of incentives for silviculture under which silvicultural performance is tied to the renewal of the industrial crown land lease (Evergreen tenure).

These changes in policy are new and still developing. Most of the strategic analyses of wood supply and consequences of silvicultural activities have been done by the provinces. Presently many major companies find themselves in a new position of:

1. Being responsible for silviculture practices, but lacking expertise in their implementation.

2. Being faced with constrained allowable cuts, and the need to re-examine the effect of improved silviculture methods upon their cut without having the same capability as the provincial landowner.

Public and professional opinion on the status of silviculture practice in Canada appear to vary widely. Professional foresters' opinion ranges from the doom and gloom "There is no hope for the industry" attitude, to the other extreme of "Dawn of a new golden era of silviculture"; the latter viewpoint usually emanating from senior public or corporate officials. Corporate advertising, both US and Canadian, appears to have strongly influenced public opinion. I am unaware of any polls of public opinion but the press clippings do reveal a wide range of viewpoint.

There is no federal or national mechanism to collect statistics on the size, cost and effectiveness of canadian silviculture programs. The annual reports of provincial government have traditionally not been very illuminating. The national assessment of the status of forest management in Canada by F.L.C. Reed and Associates (1978) and the 1979 Forestry Congress sponsored by The Canadian Pulp and Paper Association have indicated that silviculture 